\title{
Poultry to Human Passport: Cross-species Transmission of Zoonotic H7N9 Avian Influenza Virus to Humans
}

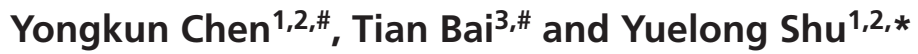

\begin{abstract}
Human infections with H7N9 avian influenza virus were first reported in the early spring of 2013, in the Yangtze-delta region of China. This virus subsequently caused five successive epidemic waves from 2013 to 2018 with highest reported cases in the last wave making this strain the most successful zoonosis influenza virus in humans in recent decades. No H7N9 human infections have been reported since 2019, probably because of the extensive vaccination of poultry. Although zoonoses of H7N9 and other subtypes of avian influenza viral infections remain rare, the virus could acquire sufficient mammalian adaptive mutations to allow it to cause a future influenza pandemic. Here, we summarize the main findings on viral and host factors affecting the interspecies transmission of the H7N9 avian influenza virus.
\end{abstract}

Keywords: cross-species transmission, H7N9 avian influenza virus, human

\section{BACKGROUND}

The influenza A virus (IAV) has been detected in a wide range of host species, thus making it a prototype for emerging viruses with pandemic potential. Wild aquatic birds are the natural reservoirs of diverse influenza $\mathrm{A}$ viruses, and the corresponding infections are usually mild or asymptomatic. Avian influenza viruses (AIVs) can be transmitted to other species, such as domestic poultry, swine, and horses. Cross-species transmission of avian IAV to humans is rare. In the past decades, sporadic human infections with AIVs, including the H5N1, H5N6, H6N1, H7N2, H7N3, H7N4, H7N7, H7N9, H9N2, H10N7, $\mathrm{H} 10 \mathrm{~N} 8, \mathrm{H} 5 \mathrm{~N} 8$, and H10N3 subtypes, have been reported [1-3]. Many of these zoonoses are "dead end": no subsequent transmission has been observed, and only a small number of cases have been reported. However, H7N9 IAV has been responsible for the major zoonosis in recent decades (Fig 1). The first laboratory-confirmed H7N9 human case was identified in eastern China in early spring, 2013 [4]. Recent exposure to poultry or visitation of live poultry markets (LPMs), where AIV can be maintained and amplified, are the main risk factors for human infection [5]. Fortunately, LPM closures effectively decreased the incidence of human infections [6]. This virus caused five continuous epidemic waves with a total of 1,568 cases and 616 deaths (case fatality rate of 39\%). No human H7N9 cases have been reported after March 2019, probably because of the massive vaccination efforts in poultry after September 2017 in China [7]. The H7N9 influenza virus causes severe or fatal outcomes in humans but shows low

\section{Edited by:}

Ke Lan, State Key Laboratory of

Virology, Wuhan University

\section{Reviewed by:}

Hao Lei, Zhejiang University

Another reviewer chose to be

anonymous

\#These authors contributed equally to this work.

*Corresponding author:

E-mail: shuylong@mail.sysu.edu.cn (YS)

'School of Public Health (Shenzhen), Shenzhen Campus of Sun Yat-sen University, Shenzhen 518107, P.R.

China

${ }^{2}$ School of Public Health (Shenzhen) Sun Yat-sen University, Guangzhou 510006, P.R. China

${ }^{3}$ Viral Zoonoses-One Health, Heinrich Pette Institute, Leibniz Institute for

Experimental Virology, Hamburg, Germany

Received: December 72021 Revised: December 262021 Accepted: December 312021 Published Online: January 212022 


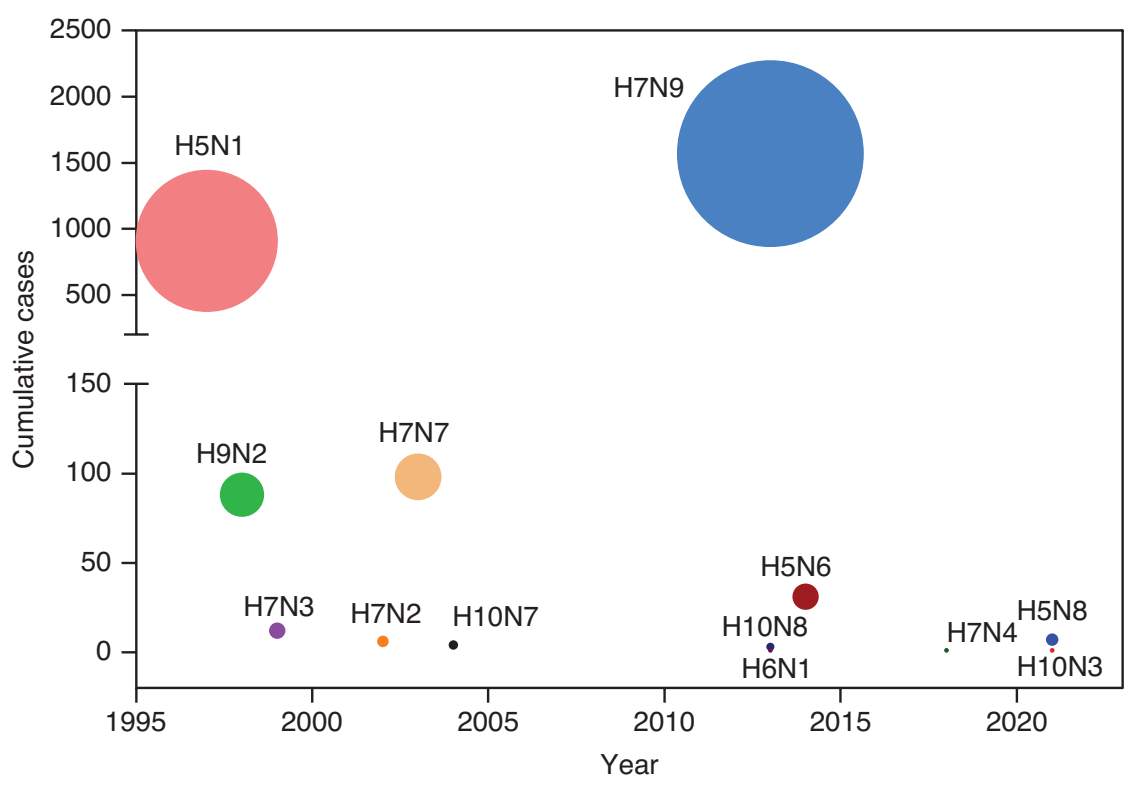

Figure 1 | Human infections with avian influenza viruses in recent decades.

The cumulative number of cases of human infection with different avian influenza virus subtypes since the first reported year or the year of a well-known epidemic (H7N7). The size of the circle represents different cumulative numbers of cases: the smallest circle represents one reported case.

pathogenicity in poultry, thus enabling "silent" evolution and transmission to humans. However, the emergence of the highly pathogenic H7N9 avian influenza (HPAI H7N9) in poultry, with increased virulence and an expanded host range to ducks, posed a new threat not only to poultry but also to public health $[8,9]$. Although no new human cases have been reported since 2019, the potential for sporadic human infections cannot be ignored, because H7N9 influenza viruses have continually been detected in poultry and related environments.

H7N9 IAVs undergo "genetic tuning" through reassortment, acquiring mutations in viral proteins that enable cross-species transmission from poultry to humans. However, human infections with the H7N9 virus remain rare, and limited human-to-human transmission has been reported; therefore, host genetic factors might also contribute to susceptibility to the H7N9 virus. Here, we summarize current findings regarding the viral and host factors that contribute to H7N9 IAV interspecies transmission.

\section{ACQUIRED FEATURES OF VIRAL PROTEINS FOR ADAPTION TO MAMMALIAN HOSTS}

H7N9 IAV is a triple-reassortant virus with two surface genes from different $\mathrm{H} 7$ and $\mathrm{N} 9$ viral subtypes, and all six internal genes from the H9N2 virus [4]. The early reassortment probably occurred in wild birds, and the second reassortment probably took place in domestic birds in east China in 2012, through exchange of internal genes from H9N2 viruses [10]. Those early strains underwent continual reassortment, and acquired amino acid substitutions in their viral proteins and features required for adaptation to mammalian hosts, such as greater binding affinity to "human-type" receptors. However, interspecies transmission from poultry to humans is not determined solely by the enhanced receptor binding ability in the cell-attachment process but is also associated with other altered viral-protein molecular markers, which might affect the transmission and replication of H7N9 influenza viruses.

\section{H7N9 IAV enhanced "human-type" receptor binding properties}

Host-cell attachment is the initial host-species barrier that can control or limit infection. The hemagglutinin (HA) protein, expressed on the surfaces of influenza viruses, binds terminal sialic acids (SAs), which are associated with the larger glycans on the surfaces of host cells, thus enabling viral internalization. In humans, $\alpha 2,6$-linked SAs are abundantly expressed in the upper respiratory tract (i.e. the nasopharynx), whereas a mixture of $\alpha 2,3$ - and $\alpha 2,6-$ linked SAs are present in the lower respiratory tract (i.e., the lungs and bronchi) [11,12]. Acquisition of a binding preference for $\alpha 2,6-$ linked SA (the "human-type" receptor) in the HA protein is believed to be a fundamental feature underlying the adaptation of AIVs to mammalian hosts. As previously reported in various influenza viruses subtypes [13-15], the G186V and Q226L mutations (H3 numbering) in the HA receptor-binding site have been detected in H7N9 influenza viruses, and contribute to its enhanced "human-type" receptor binding preference [16]. Furthermore, substitutions of both G186V and Q226L, or G186V alone, have been detected in the HPAI H7N9 virus $[17,18]$.

Neuraminidase is a sialidase protein that cleaves SA from infected cell surfaces, thus enabling the release of progeny virions for viral spreading [19]. The N9 of H7N9 viruses bears a short-stalk with a deletion of 5 amino acids at 
positions 69-73, which is commonly observed in terrestrial poultry [20]. Furthermore, N9 shows inefficient cleavage of $\alpha 2,6$-linked SA receptors, thus potentially compromising aerosol transmission in ferrets [21]. However, a recent study has indicated that N9 also has receptor binding properties mediated by a hemadsorption site in addition to an enzymatic site with enhanced binding to "human-type" receptors [22].

Therefore, the overall increase in $\alpha 2,6$-linked SA-receptor binding properties due to mutations in the HA protein as well as the additional neuraminidase-binding feature are possible advantages that may facilitate H7N9 influenza virus infection in human cells and mammalian hosts. Unlike H5N1 IAV, which has a binding preference for $\alpha 2,3$-linked SA (the "avian-type" receptor) and has only rarely cased human infections since 1997, H7N9 influenza virus has an enhanced $\alpha 2,6$-linked SA binding specificity, which might partially explain the larger number of human cases caused by this novel avian influenza virus [16]. H7N9 influenza viruses have acquired an ability to bind "human-type" receptors. However, they have retained their ability to bind $\alpha 2,3$-linked SA [23]. Furthermore, N9 shows poor cleavage activity toward $\alpha 2,6$-linked SA receptors, thus potentially limiting viral spread and release from human cell surfaces for further replication. These characteristics may explain why only limited human-to-human transmission has been reported $[5,24,25]$ and why efficient airborne transmission of the H7N9 virus has not been observed in ferret models [26].

\section{H7N9 IAV enhances replication or virulence in mammalian hosts}

The genomic replication of the avian influenza virus is poor in mammalian cells. However, multiple molecular viral polymerase mutations that overcome this major species barrier have been reported. Polymerase basic protein 2 (PB2) is a major host determinant, and T271A, Q591K, E627K, and D701N substitutions have been reported in most LPAI and HPAI human H7N9 isolates $[18,20]$. Those mutations are associated with greater polymerase activity in mammalian cells or enhanced virulence in mice. The E627K or D701N mutations in PB2 are critical for the adaptation of AIVs to mammalian hosts [27-29]. These mutations increase the polymerase activity and viral replication of $\mathrm{H} 7 \mathrm{~N} 9$ viruses in vitro and in vivo [30-32]. Although no human isolates with dual mutations in PB2 at positions 627 and 701 have been reported, these mutations have been discovered in a ferret transmission model and have also been found to confer enhanced fitness in mammalian cells and mice [33]. Another subunit of the polymerase basic protein $\mathrm{PB} 1$ has a potentially biologically important I $368 \mathrm{~V}$ mutation $[34,35]$, which is associated with acquired transmissibility of the H5N1 avian influenza virus among ferrets [36]. The PB1 gene also encodes the full-length PB1-F2 protein of approximately 89 amino acids. However, some H7N9 isolates encode only shorter-length PB1-F2 proteins, as also observed in other influenza A viruses [20]. This shorter length is associated with greater pathogenicity in mice, but its contribution to the virulence of $\mathrm{H} 7 \mathrm{~N} 9$ viruses remains unclear. Moreover, human-like signatures have been reported, including PA V100A, K356R, and S409N mutations [20].

\section{HOST FACTORS CONTRIBUTING TO SUSCEPTIBILITY TO H7N9 INFECTION}

\section{Human genetic determinants of H7N9 avian influenza viral infections}

Epidemiological investigations have revealed that exposure to poultry is the most likely independent risk factor for human H7N9 infections. In one study, 73\% (887/1220) of infected patients were found to have been exposed to infected poultry and/or contaminated environments, mainly LPMs, whereas only $7 \%(91 / 1220)$ of the confirmed cases were associated with occupational exposure to poultry [37]. Furthermore, serological surveys of poultry workers have indicated undetectable or limited neutralizing antibodies against the H7N9 virus (seropositivity rate of $0.11-0.93 \%$ ) $[38,39]$. Additionally, in the general population, two sero-epidemiologic surveys between 2013 and 2016 have reported zero or very low seropositivity rates for $\mathrm{H} 7 \mathrm{~N} 9$ influenza virus $[38,40]$. Because human infections with $\mathrm{H} 7 \mathrm{~N} 9$ viruses remain very rare, host genetic factors might also determine susceptibility to H7N9 infections.

Previous studies have shown that host genetic factors are important in the pathogenesis of, or susceptibility to, influenza viral infections [41]. Single-nucleotide variants (SNVs) of several genes, including TMPRSS2, IFITM3, TLR3, and CD55, are associated with the severity of human infections with H7N9 viruses [42-44] (Table 1). The rs4820294/rs2899292 haplotype GG of LGALS1 is associated with protection from H7N9 infections, possibly because of significantly higher LGALS1 mRNA and protein expression in lymphoblast cell lines [45]. However, little is known regarding genetic predisposition to H7N9 infection. In addition, most SNVs identified in single-variant analyses have high minor-allele frequencies (MAF $>5 \%$, gnomAD) in humans. Furthermore, with the development of next-generation sequencing, rare SNVs predisposing humans to H7N9 infection can be identified through human whole-genome sequencing. For example, a low-frequency variant of UBXN11 and three HLA alleles (DQB1*06:01, DQA1*05:05, and $C \star 12: 02)$ have been associated with H7N9 infection in human [46]. Moreover, our recent investigation of the contribution of rare mutations in poultry workers frequently exposed to H7N9 viruses, compared with other populations, has identified 17 defective SNVs in the myxovirus resistance 1 (MX1) locus in H7N9-infected patients, without sex differences, on the basis of whole-genome sequencing [47]. In vitro experiments also demonstrated that 14 of the $17 \mathrm{MxA}$ protein variants, including H7N9, H7N7, and H5N1, had lost the ability to inhibit avian IAV replication. These results demonstrate the key role of MX1-based antiviral defense in controlling interspecies transmission. Additionally, previous studies have shown that human MxA inhibits influenza virus replication 
TABLE 1 | Host genes associated with human susceptibility to H7N9 infections.

\begin{tabular}{|c|c|c|c|c|}
\hline Host gene & Single-nucleotide variant (rsID) & Variant class & Animal studies & Reference(s) \\
\hline IFITM 3 & rs12252 & $\begin{array}{l}\text { Common variants } \\
(\mathrm{MAF}>5 \%)\end{array}$ & + & {$[43,44]$} \\
\hline TMPRSS2 & rs2070788/rs383510 & $\begin{array}{l}\text { Common variants } \\
(\mathrm{MAF}>5 \%)\end{array}$ & + & {$[42]$} \\
\hline LGALS1 & rs4820294/rs2899292 haplotype GG & $\begin{array}{l}\text { Common variants } \\
(\mathrm{MAF}>5 \%)\end{array}$ & + & {$[45]$} \\
\hline CD55 & rs2564978 & $\begin{array}{l}\text { Common variants } \\
(\mathrm{MAF}>5 \%)\end{array}$ & - & [43] \\
\hline UBXN11 & rs189256251 & $\begin{array}{l}\text { Low-frequency variants } \\
(0.5 \%<\text { MAF < } \% \text { ) }\end{array}$ & - & {$[46]$} \\
\hline$M \times 1$ & $\begin{array}{l}\text { c. } 88 C>T \\
\text { rs138644617 } \\
\text { rs764277171 } \\
\text { rs368357662 } \\
\text { rs141166870 } \\
\text { rs779947445 } \\
\text { rs762946488 } \\
\text { rs754725725 } \\
\text { c. } 439 C>A \\
\text { c.757G >A } \\
\text { rs1172769444 } \\
\text { rs370594768 } \\
\text { c. } 1589 T>G \\
\text { c. } 1625 T>C\end{array}$ & $\begin{array}{l}\text { Rare variants } \\
(\mathrm{MAF}<0.5 \%)\end{array}$ & + & {$[47,55]$} \\
\hline
\end{tabular}

MAF, minor-allele frequency in East Asian individuals in the Genome Aggregation Database v2.2.2 (gnomAD); (+) validated and (-) not validated in mouse models.

by blocking the nuclear translocation of incoming viral ribonucleoproteins with the help of additional cofactors to achieve anti-influenza activity [48]. To verify the antiviral mechanism of human MxA and how missense mutations in different domains influence its antiviral effects, further studies are needed. Such studies would improve understanding of how AIVs cross species barriers.

\section{Male sex bias of H7N9 infection}

Interestingly, men have consistently accounted for approximately $70 \%$ of all H7N9 infection cases in the past five epidemic waves [37]. Previously, differential healthcare-seeking/access behavior between men and women was proposed to contribute to sex biases in the case distributions in surveillance and diagnosis [49]. However, a modeling study has revealed that increased exposure time in LPMs does not increase the risk of H7N9 infections in older men, thus suggesting that underlying biological host factors between sexes may influence the susceptibility and severity differences in H7N9 infections [50]. Interestingly, a mouse model has indicated that female mice have greater weight loss and lower survival rates than male mice after H7N9 infection [51]. Although no sex-specific differences in viral titers have been observed in the lung, female mice show a greater inflammatory response than male mice. Similarly, observations regarding the currently circulating SARS-CoV-2 virus also indicate the importance of sex differences: male sex is a risk factor for hospitalization [52,53]. A recent study has also identified that perturbed sex-hormone metabolism might be a hallmark of critically ill men with Coronavirus disease 2019 [54]. In contrast to the pandemic of zoonotic SARS-CoV-2 infections, to which both sexes are susceptible, H7N9 infections show no or limited human-to-human transmission, but display a very apparent bias toward males. Hence, further investigation of the underlying mechanisms of sex-dependent disease outcomes of H7N9 infections is needed to guide patient-centered clinical management.

\section{CONCLUSIONS}

Studies have revealed mutations in viral proteins that overcome the host barrier and host factors that increase the susceptibility to H7N9 infections in humans (Fig 2). Although no H7N9 human cases have been reported, this virus is still occasionally detected in poultry or contaminated environments. Further viral adaptations, such as stronger humanreceptor binding ability, may still pose a threat for a future pandemic, and individuals with susceptible host genes may develop severe outcomes after infection. Many scientific questions remain to be addressed. For example, what are the underlying mechanisms of male-biased H7N9 infections? What are the potential mechanisms of discovered host genes, such as MX1? Understanding the mechanisms of viral adaptive changes and host susceptibility might provide new potential targets for better, more precise preparation for future zoonoses leading to epidemics or pandemics. 


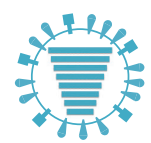

Avian influenza A H7N9 virus

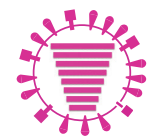

Human-adapted influenza A H7N9 virus

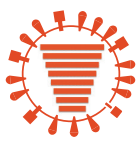

More human adaptive Mutations

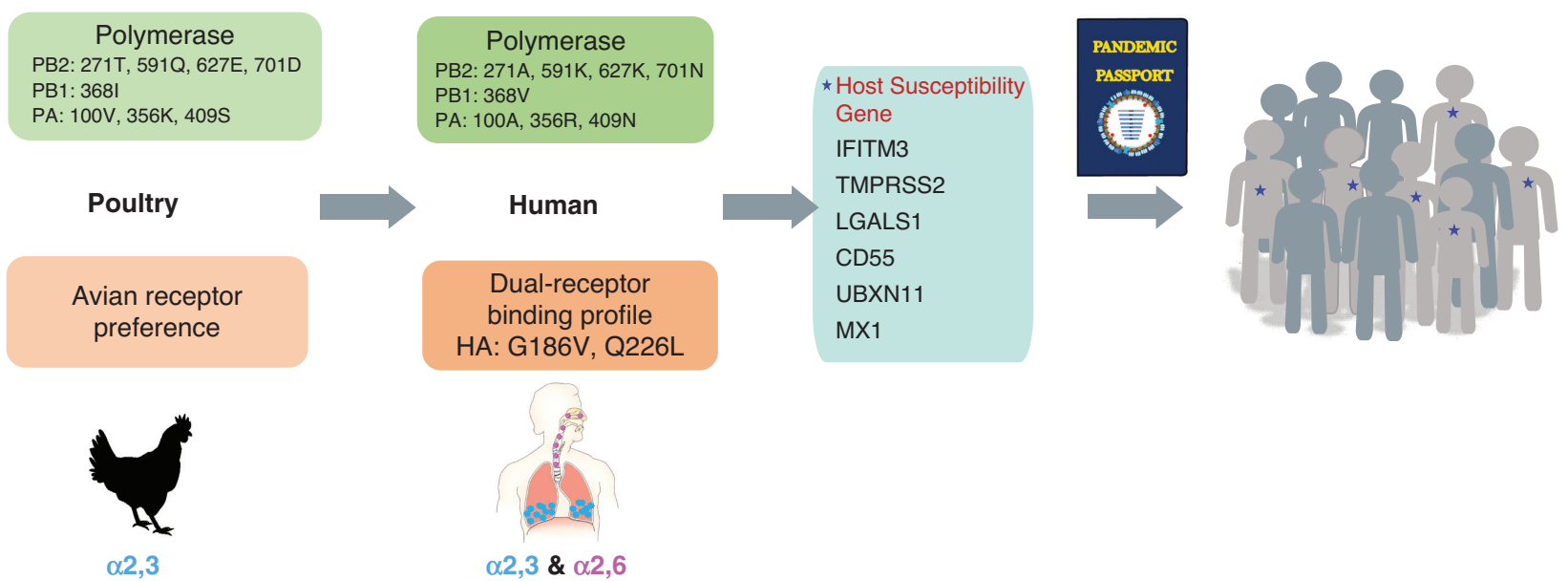

Figure 2 | Viral and host factors overcome the cross-species barrier in human H7N9 infections.

Mutations in viral proteins and host factors shown to increase susceptibility to H7N9 infections in humans. Further adaptation of the H7N9 virus poses a continued pandemic threat. The presence of susceptible host genes may be associated with poorer disease outcomes.

\section{FUNDING}

This work was supported by grants from the National Key Research and Development Program of China (grant Nos. 2021YFC2300102 and 2021YF(2300103), the Shenzhen Science and Technology Program (grant No. KQTD20180411143323605), the National Natural Science Foundation of China (grant No. 81961128002), and the Guangdong Province Science and Technology Innovation Strategy Special Fund (grant No. 2018A030310337).

\section{REFERENCES}

1. Shi W, Gao GF. Emerging H5N8 avian influenza viruses. Science. 2021;372(6544):784-786.

2. Jing J, Wang L, Wang G, Dai Z, Ren W, Yi C, et al. A human infection case with avian origin H10N3 influenza virus. Quant Imaging Med Surg. 2021;11(10):4508-4510.

3. Wang D, Zhu W, Yang L, Shu Y. The epidemiology, virology, and pathogenicity of human infections with avian influenza viruses. Cold Spring Harb Perspect Med. 2021;11(4):a038620.

4. Gao R, Cao B, Hu Y, Feng Z, Wang D, Hu W, et al. Human infection with a novel avian-origin influenza $A(H 7 N 9)$ virus. N Engl J Med. 2013;368(20):1888-1897.

5. Li Q, Zhou L, Zhou M, Chen Z, Li F, Wu H, et al. Epidemiology of human infections with avian influenza $\mathrm{A}(\mathrm{H} 7 \mathrm{~N} 9)$ virus in China. N Engl J Med 2014;370(6):520-532.

6. Yu H, Wu JT, Cowling BJ, Liao Q, Fang VJ, Zhou S, et al. Effect of closure of live poultry markets on poultry-to-person transmission of avian influenza A H7N9 virus: an ecological study. Lancet. 2014;383(9916):541-548.

7. Zeng X, Tian G, Shi J, Deng G, Li C, Chen H. Vaccination of poultry successfully eliminated human infection with H7N9 virus in China. Sci China Life Sci. 2018;61(12):1465-1473.

8. Ke C, Mok CKP, Zhu W, Zhou H, He J, Guan W, et al. Human infection with highly pathogenic Avian influenza $A(H 7 N 9)$ virus, China. Emerg Infect Dis. 2017;23(8):1332-1340.

9. Shi J, Deng G, Ma S, Zeng X, Yin X, Li M, et al. Rapid evolution of H7N9 highly pathogenic viruses that emerged in china in 2017. Cell Host Microbe. 2018;24(4):558-568.e557.
10. Wu A, Su C, Wang D, Peng Y, Liu M, Hua S, et al. Sequential reassortments underlie diverse influenza H7N9 genotypes in China. Cell Host Microbe. 2013;14(4):446-452.

11. Walther T, Karamanska R, Chan RW, Chan MC, Jia N, Air G, et al. Glycomic analysis of human respiratory tract tissues and correlation with influenza virus infection. PLoS Pathog. 2013;9(3):e1003223.

12. Chandrasekaran A, Srinivasan A, Raman R, Viswanathan K, Raguram S, Tumpey T M, et al. Glycan topology determines human adaptation of avian H5N1 virus hemagglutinin. Nat Biotechnol. 2008;26(1):107-113.

13. Yamada S, Suzuki Y, Suzuki T, Le MQ, Nidom CA, Sakai-Tagawa $Y$, et al. Haemagglutinin mutations responsible for the binding of H5N1 influenza A viruses to human-type receptors. Nature. 2006;444(7117):378-382.

14. Matrosovich M, Tuzikov A, Bovin N, Gambaryan A, Klimov $A$, Castrucci MR, et al. Early alterations of the receptorbinding properties of $\mathrm{H} 1, \mathrm{H} 2$, and $\mathrm{H} 3$ avian influenza virus hemagglutinins after their introduction into mammals. J Virol. 2000;74(18):8502-8512.

15. Srinivasan $K$, Raman $R$, Jayaraman A, Viswanathan $K$, Sasisekharan R. Quantitative description of glycan-receptor binding of influenza $\mathrm{A}$ virus $\mathrm{H} 7$ hemagglutinin. PLoS One. 2013;8(2):e49597.

16. Zhou J, Wang D, Gao R, Zhao B, Song J, Qi X, et al. Biological features of novel avian influenza A (H7N9) virus. Nature. 2013;499(7459):500-503.

17. Yang L, Zhu W, Li X, Chen M, Wu J, Yu P, et al. Genesis and spread of newly emerged highly pathogenic H7N9 avian viruses in mainland China. J Virol. 2017;91(23):e01277-17.

18. Zhu W, Zhou J, Li Z, Yang L, Li X, Huang W, et al. Biological characterisation of the emerged highly pathogenic avian influenza (HPAl) $A(\mathrm{H} 7 \mathrm{~N} 9)$ viruses in humans, in mainland China, 2016 to 2017. Euro Surveill. 2017;22(19):30533.

19. Long J S, Mistry B, Haslam S M, Barclay WS. Host and viral determinants of influenza A virus species specificity. Nat Rev Microbiol. 2019;17(2):67-81.

20. Wang D, Yang L, Gao R, Zhang X, Tan Y, Wu A, et al. Genetic tuning of the novel avian influenza $A(H 7 N 9)$ virus 
during interspecies transmission, China, 2013. Euro Surveill. 2014;19(25):20836.

21. Blumenkrantz D, Roberts KL, Shelton $H$, Lycett $S$, Barclay WS. The short stalk length of highly pathogenic avian influenza H5N1 virus neuraminidase limits transmission of pandemic H1N1 virus in ferrets. J Virol. 2013;87(19):10539-10551.

22. Benton DJ, Wharton SA, Martin SR, McCauley JW. Role of neuraminidase in influenza $A(H 7 N 9)$ virus receptor binding. J Virol. 2017;91(11):e02293.

23. Ramos I, Krammer F, Hai R, Aguilera D, Bernal-Rubio D, Steel J, et al. H7N9 influenza viruses interact preferentially with alpha2,3-linked sialic acids and bind weakly to alpha2,6-linked sialic acids. J Gen Virol. 2013;94(Pt 11):2417-2423.

24. Hu J, Zhu Y, Zhao B, Li J, Liu L, Gu K, et al. Limited human-tohuman transmission of avian influenza $A(H 7 N 9)$ virus, Shanghai, China, March to April 2013. Euro Surveill. 2014;19(25):20838.

25. Liu B, Havers FP, Zhou L, Zhong H, Wang X, Mao S, et al. Clusters of human infections with avian influenza $A(H 7 N 9)$ virus in China, March 2013 to June 2015. J Infect Dis. 2017;216(Suppl 4):S548-S554.

26. Richard M, Schrauwen EJ, de Graaf M, Bestebroer TM, Spronken MI, van Boheemen S, et al. Limited airborne transmission of H7N9 influenza A virus between ferrets. Nature. 2013;501(7468):560-563.

27. Hatta M, Gao P, Halfmann P, Kawaoka Y. Molecular basis for high virulence of Hong Kong H5N1 influenza A viruses. Science. 2001;293(5536):1840-1842.

28. Gabriel G, Dauber B, Wolff T, Planz O, Klenk HD, Stech J. The viral polymerase mediates adaptation of an avian influenza virus to a mammalian host. Proc Natl Acad Sci U S A. 2005;102(51):18590-18595.

29. Subbarao EK, London W, Murphy BR. A single amino acid in the PB2 gene of influenza A virus is a determinant of host range. J Virol. 1993;67(4):1761-1764.

30. Mok CK, Lee HH, Lestra M, Nicholls JM, Chan MC, Sia SF, et al. Amino acid substitutions in polymerase basic protein 2 gene contribute to the pathogenicity of the novel A/H7N9 influenza virus in mammalian hosts. J Virol. 2014;88(6):3568-3576.

31. Yamayoshi S, Fukuyama S, Yamada S, Zhao D, Murakami S, Uraki $\mathrm{R}$, et al. Amino acids substitutions in the PB2 protein of $\mathrm{H} 7 \mathrm{~N} 9$ influenza A viruses are important for virulence in mammalian hosts. Sci Rep. 2015;5:8039.

32. Zhang H, Li X, Guo J, Li L, Chang C, Li Y, et al. The PB2 E627K mutation contributes to the high polymerase activity and enhanced replication of H7N9 influenza virus. J Gen Virol. 2014;95(Pt 4):779-786.

33. Zhu W, Li L, Yan Z, Gan T, Li L, Chen R, et al. Dual E627K and D701N mutations in the PB2 protein of $A(H 7 N 9)$ influenza virus increased its virulence in mammalian models. Sci Rep. 2015;5:14170.

34. Zhu W, Zou X, Zhou J, Tang J, Shu Y. Residues $41 \mathrm{~V}$ and/or $210 \mathrm{D}$ in the NP protein enhance polymerase activities and potential replication of novel influenza (H7N9) viruses at low temperature. Virology J. 2015;12:71.

35. Zhu W, Shu Y. Genetic tuning of avian influenza A (H7N9) virus promotes viral fitness within different species. Microbes Infec./ Institut Pasteur. 2015;17(2):118-122.

36. Herfst $S$, Schrauwen EJ, Linster M, Chutinimitkul S, de Wit E, Munster VJ, et al. Airborne transmission of influenza A/H5N1 virus between ferrets. Science. 2012;336(6088):1534-1541.

37. Wang $X$, Jiang $H$, Wu $P$, Uyeki TM, Feng $L$, Lai $S$, et al. Epidemiology of avian influenza A H7N9 virus in human beings across five epidemics in mainland China, 2013-17: an epidemiological study of laboratory-confirmed case series. Lancet Infecti Dis. 2017;17(8):822-832.
38. Xiang N, Bai T, Kang K, Yuan H, Zhou S, Ren R, et al. Seroepidemiologic study of influenza A(H7N9) infection among exposed populations, China 2013-2014. Influenza Other Respir Viruses. 2017;11(2):170-176.

39. Ma MJ, Zhao T, Chen SH, Xia X, Yang XX, Wang GL, et al. Avian influenza $A$ virus infection among workers at live poultry markets, China, 2013-2016. Emerg Infect Dis. 2018;24(7):1246-1256.

40. Bai T, Zhou J, Shu Y. Serologic study for influenza A (H7N9) among high-risk groups in China. N Engl J Med. 2013;368(24):2339-2340.

41. Clohisey S, Baillie JK. Host susceptibility to severe influenza A virus infection. Crit Care. 2019;23(1):303.

42. Cheng Z, Zhou J, To KK, Chu H, Li C, Wang D, et al. Identification of TMPRSS2 as a susceptibility gene for severe 2009 pandemic $A(H 1 N 1)$ influenza and $A(H 7 N 9)$ influenza. J Infect Dis. 2015;212(8):1214-1221.

43. Lee N, Cao B, Ke C, Lu H, Hu Y, Tam CHT, et al. IFITM3, TLR3, and CD55 gene SNPs and cumulative genetic risks for severe outcomes in Chinese patients with H7N9/H1N1pdm09 influenza. J Infect Dis. 2017, 216(1):97-104.

44. Wang Z, Zhang A, Wan Y, Liu X, Qiu C, Xi X, et al. Early hypercytokinemia is associated with interferon-induced transmembrane protein-3 dysfunction and predictive of fatal H7N9 infection. Proc Natl Acad Sci U S A. 2014;111(2):769-774.

45. Chen Y, Zhou J, Cheng Z, Yang S, Chu H, Fan Y, et al. Functional variants regulating LGALS1 (Galectin 1) expression affect human susceptibility to influenza A(H7N9). Sci Rep. 2015;5:8517.

46. Zhao B, Chen Y, Li M, Zhou J, Teng Z, Chen J, et al. Novel susceptibility loci for $A(H 7 N 9)$ infection identified by next generation sequencing and functional analysis. Sci Rep. 2020;10(1):11768.

47. Chen Y, Graf L, Chen T, Liao Q, Bai T, Petric PP, et al. Rare variant MX1 alleles increase human susceptibility to zoonotic H7N9 influenza virus. Science. 2021;373(6557):918-922.

48. Haller $O$, Kochs $G$. Mx genes: host determinants controlling influenza virus infection and trans-species transmission. Hum Genet. 2020;139(6-7):695-705.

49. Arima Y, Vong S, World Health Organization Outbreak Response Team. Human infections with avian influenza $A(H 7 N 9)$ virus in China: preliminary assessments of the age and sex distribution. Western Pac Surveill Response J. 2013;4(2):1-3.

50. Rivers C, Lum K, Lewis B, Eubank S. Estimating human cases of avian influenza $A(H 7 N 9)$ from poultry exposure. PLoS Curr. 2013;5.

51. Hoffmann J, Otte A, Thiele S, Lotter H, Shu Y, Gabriel G. Sex differences in H7N9 influenza A virus pathogenesis. Vaccine. 2015;33(49):6949-6954.

52. Williamson EJ, Walker AJ, Bhaskaran K, Bacon S, Bates C, Morton CE, et al. Factors associated with COVID-19-related death using OpenSAFELY. Nature. 2020;584(7821):430-436.

53. Peckham H, de Gruijter NM, Raine C, Radziszewska A, Ciurtin C, Wedderburn LR, et al. Male sex identified by global COVID19 meta-analysis as a risk factor for death and ITU admission. Nat Commun. 2020;11(1):6317.

54. Schroeder M, Schaumburg B, Mueller Z, Parplys A, Jarczak D, Roedl $K$, et al. High estradiol and low testosterone levels are associated with critical illness in male but not in female COVID19 patients: a retrospective cohort study. Emerg Microbes Infect. 2021;10(1):1807-1818.

55. Deeg C, Mutz P, Kallfass C, Nurnberger C, Soubies S, Staeheli P. Transgenic mice carrying interferon-regulated human MxA locus are highly resistant to avian but not human influenza A viruses. Cytokine. 2013;63(3):258-258. 


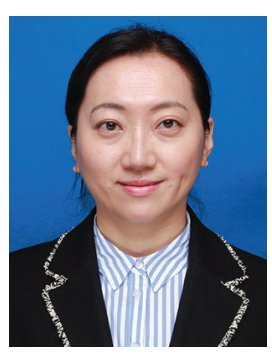

Dr. Yongkun Chen finished her PhD in 2017, at the National Institute for viral disease control and prevention, China CDC. After that she joined the School of Public Health (Shenzhen), Sun Yat-sen University, as an associate researcher. Her research focuses on host genetic factors associated with clinical severity and susceptibility of zoonotic influenza viruses caused human infections. Recently she identified the rare mutations in MX1 gene abrogated the innate barrier that normally prevents zoonotic H7N9 IAV infections, and thus increased the susceptibility of human infections with H7N9 avian influenza virus (Chen et al, Science, 2021). 